\title{
Prediction of cognitive impairment via deep learning trained with multi-center neuropsychological test data
}

Min Ju Kang ${ }^{1,2}$, Sang Yun Kim ${ }^{1}$, Duk L. Na ${ }^{3}$, Byeong C. Kim, Dong Won Yang ${ }^{5}$, Eun-Joo Kim ${ }^{6}$, Hae Ri Na ${ }^{7}$, Hyun Jeong Han ${ }^{8}$, Jae-Hong Lee ${ }^{9}$, Jong Hun Kim ${ }^{10}$, Kee Hyung Park ${ }^{11}$, Kyung Won Park ${ }^{12}$, Seol-Heui Han ${ }^{13}$, Seong Yoon Kim ${ }^{14}$, Soo Jin Yoon ${ }^{15}$, Bora Yoon ${ }^{16}$, Sang Won Seo ${ }^{3}$, So Young Moon ${ }^{17}$, YoungSoon Yang ${ }^{2}$, Yong S. Shim ${ }^{18}$, Min Jae Baek ${ }^{1}$, Jee Hyang Jeong ${ }^{19}$, Seong Hye Choi ${ }^{20}$ and Young Chul Youn ${ }^{21^{*}}$ (D

\begin{abstract}
Background: Neuropsychological tests (NPTs) are important tools for informing diagnoses of cognitive impairment (CI). However, interpreting NPTs requires specialists and is thus time-consuming. To streamline the application of NPTs in clinical settings, we developed and evaluated the accuracy of a machine learning algorithm using multicenter NPT data.

Methods: Multi-center data were obtained from 14,926 formal neuropsychological assessments (Seoul Neuropsychological Screening Battery), which were classified into normal cognition (NC), mild cognitive impairment (MCl) and Alzheimer's disease dementia (ADD). We trained a machine learning model with artificial neural network algorithm using TensorFlow (https://www.tensorflow.org) to distinguish cognitive state with the 46-variable data and measured prediction accuracies from 10 randomly selected datasets. The features of the NPT were listed in order of their contribution to the outcome using Recursive Feature Elimination.
\end{abstract}

Results: The ten times mean accuracies of identifying $\mathrm{Cl}(\mathrm{MCl}$ and $\mathrm{ADD})$ achieved by $96.66 \pm 0.52 \%$ of the balanced dataset and $97.23 \pm 0.32 \%$ of the clinic-based dataset, and the accuracies for predicting cognitive states (NC, MCl or ADD) were $95.49 \pm 0.53$ and $96.34 \pm 1.03 \%$. The sensitivity to the detection $\mathrm{Cl}$ and $\mathrm{MCl}$ in the balanced dataset were 96.0 and 96.0\%, and the specificity were 96.8 and $97.4 \%$, respectively. The 'time orientation' and '3-word recall' score of MMSE were highly ranked features in predicting $\mathrm{Cl}$ and cognitive state. The twelve features reduced from 46 variable of NPTs with age and education had contributed to more than $90 \%$ accuracy in predicting cognitive impairment.

Conclusions: The machine learning algorithm for NPTs has suggested potential use as a reference in differentiating cognitive impairment in the clinical setting.

Keywords: Machine learning, Neuropsychological test, Dementia, Mild cognitive impairment, Alzheimer's disease

\section{Background}

Cognitive impairment is a spectrum that ranges from subjective cognitive decline to mild cognitive impairment (MCI) and - at its end - dementia [1]. The diagnosis of MCI and Alzheimer's disease dementia (ADD) depends on the clinical decision by clinicians, where neuropsychological tests help inform the presence of objective cognitive impairment [2-5].

\footnotetext{
*Correspondence: neudoc@cau.ac.kr

${ }^{21}$ Department of Neurology, College of Medicine, Chung-Ang University, Seoul, South Korea

Full list of author information is available at the end of the article
}

However, assessing individual cognitive states using neuropsychological test (NPT) is time-consuming, as it requires the evaluation of an extensive amount of information $[6,7]$; this is in part due to the accuracy and efficiency of NPTinformed diagnosis being determined by the level of practitioner expertise.

The advent of machine learning algorithms that can analyze complex medical data may streamline the application of NPT [8, 9]. An algorithm learns the relationship between the input data (test score) and the corresponding output variables (clinical diagnosis). Once 
the learning process is completed, the algorithm can yield classifications or predictions when new data is inputted [10]. Several studies have applied machine learning to the differential diagnosis of dementia: Gurevich used Consortium to Establish a Registry for Alzheimer's disease (CERAD) to identify Alzheimer's disease (AD) among 158 subjects based on cerebral spinal fluid biomarkers and thereby achieved a classification accuracy of $89 \%$ [11]; and using a cohort of 272 subjects, Weakley et al. applied machine learning to 27 measures of NPT to yield classifications of clinical-dementia ratings. They also used machine learning to explore the configuration of measures for variable reduction and achieved an efficient predictive model using a maximum of six variables [12]. However, such investigations are among a hitherto limited effort to apply machine learning to the diagnosis and prognostic estimation of cognitive decline, and studies benefitting from large datasets are unavailable. Recently, some researchers found that some MRI and/or NPT features can be used to predict AD conversion using machine learning [13]. Although there was limited number of subjects, they used well stratified randomized dataset.

Research on screening cognitive impairment using the machine learning algorithm published by Youn et al. is similar in that it predicts cognitive impairment [14]. However, it is designed to evaluate the accuracy of a Logistic Regression algorithm based on Mini-mental status examination and simple questionnaire for screening purposes, which would be valuable in primary health care. Unlike the previous study, this work is intended to predict cognitive disorders using formal neuropsychological tests conducted by patients at hospitals, suggesting the possibility of reducing evaluators' loads.

The Seoul Neuropsychological Screening Battery (SNSB) has been widely used for the assessment of cognitive functioning in patients with neurological disorders in Korea. The SNSB includes measures for attention, language, visuospatial function, memory and frontal executive function [15-18]. Using the SNSB, the present study applied machine learning algorithms to data on 46 variables collected from 17,139 subjects: a large set of NPT data and subjects were obtained from a well-controlled dementia cohort study $[19,20]$. We thereby aimed to develop an algorithm to efficiently conduct an NPT-informed pre-reading of cognitive impairment among patients.

\section{Methods}

The SNSB data were obtained from a study of the Clinical Research Center for Dementia of South Korea (CREDOS), memory clinics of Bundang Seoul University Hospital (BDSNUH), and Chung-Ang University Hospital (CAUH). The CREDOS study was a prospective, multi-center, hospital-based cohort study with 56 participating hospitals and was designed to assess the occurrence and risk factors of cognitive disorders [19-22]. The SNSB test was conducted by trained psychologists, and at the beginning of the CREDOS study, four workshop were held for psychiatrists or neurologists to increase the diagnostic concordance. Subjects complaining of memory lapses were clinically classified into normal cognition (NC), $\mathrm{MCI}$, and $\mathrm{AD}$ dementia (ADD) by dementia-special clinicians based on the CREDOS criteria [14, 19, 20,23].

A total of 17,139 subjects (10,178 from CRCD, 4210 from BDSNUH, and 2751 from CAUH) were recruited. We excluded 2213 subjects for whom no final diagnosis was available or who had severe white matter hyperintensities (deep white matter hyperintensity $\geq 25 \mathrm{~mm}$ ) [19, 21, 24]; eligible subjects thus totaled to 14,926: 3217 had NC (21.55\%), 6002 had MCI (40.21\%), and 5707 had ADD (38.24\%): "Clinic-based dataset". The dataset was balanced by using "random.sample" method of python 3.6 through random under-sampling the majority group at nearly same to the NC: "Balanced dataset" (Fig. 1). The balanced 2-way classification dataset composed $3217 \mathrm{NC}$ and $3231 \mathrm{CI}$, and 3-way classification dataset composed $3217 \mathrm{NC}$ and $3217 \mathrm{MCI}$ and $3235 \mathrm{ADD}$. This study was approved by the institutional review boards of the participating centers (IRB number C2012049(744)).

The features from SNSB data were $46+1$ variables, including one target outcome (Table 1). Variables of ratio scale used raw data obtained from the subjects, and ordinal scale were shown as $0,1,2$, and 3 , respectively; 0 represents subject could not perform the task at all, 1 was abnormal, 2 was borderline and 3 was normal. The variables of ordinal scale were marked with "(o)" in Table 1 . The target outcome was "clinical diagnosis" composed of participants falling within one of three diagnostic classes: NC, MCI, or ADD. We trained a machine learning algorithm using TensorFlow (https:// www.tensorflow.org) to distinguish the states of the subjects based on the SNSB data [25].

\section{Test a. differentiate subjects with Normal cognition and cognitive impairment (Additional file 1: Table S3)}

Using the two type of dataset ("clinic-based dataset" and "balance dataset") in which the subjects were divided into the two groups of $\mathrm{NC}$ and cognitive impairment (CI), which included MCI and ADD, we developed an algorithm to predict for cognitive impairment (2-way classification).

The first step in modeling the algorithm requires the dataset to go through the following pre-processing steps. We imported the data formatted with '.csv' and used the train_test_split function from scikit-learn library (https://scikit-learn.org/) to randomly split them into training and test datasets. The train_size was 0.75 , which indicated the percentage of the data to be withheld for 


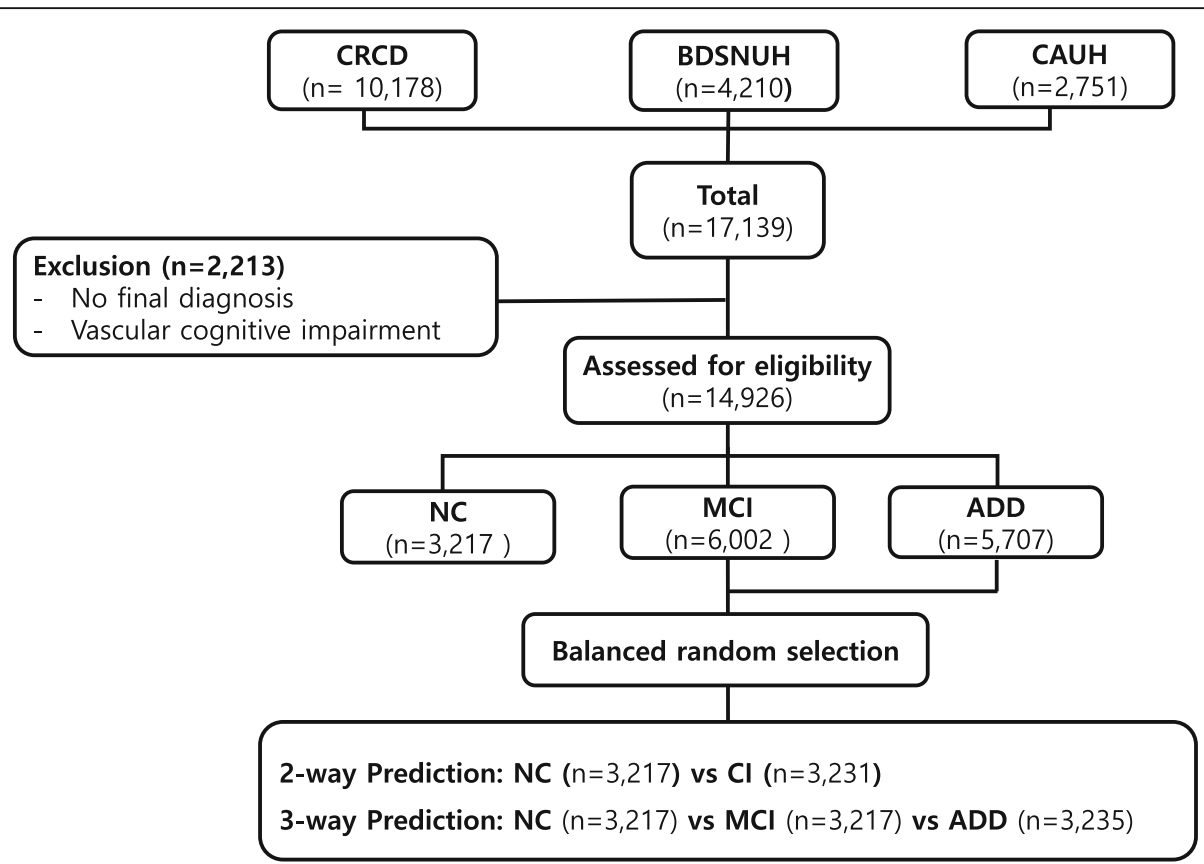

Fig. 1 Enrollment for SNSB machine-learning analysis. CRCD, Clinical Research Center for Dementia of Korea; BDSNUH, Bungdang Seoul National University Hospital; CAUH, Chung-Ang University Hospital; NC, Normal Cognition; MCI, Mild Cognitive Impairment; ADD, Alzheimer's

Disease Dementia

training; the test dataset was thus comprised of the remaining $25 \%$ of the data. Every score of features was normalized with mean and standard deviation.

The training dataset was used for further model training via TensorFlow, a commonly used open-source software library for machine learning developed by Google based on python [25]. Although it is an algorithm that differentiate subjects with $\mathrm{CI}$ from $\mathrm{NC}$, 'one_hot encoding' was used

Table 1 List of 46 features from Seoul Neuropsychological Screening Battery test

1.Education duration, 2.Age, 3.Digit span Forward, 4.Digit span Backward, 5.Letter cancellation (0), 6.Spontaneous speech fluency(o),

7.Spontaneous speech contents(0), 8.Comprehension(0), 9.Naming KBNT, 10.Finger naming(o), 11.Right left orientation(o), 12.Body part identification(o), 13.Praxis Ideomotor(o), 14.Praxis buccofacial(o), 15.Calculation total score, 16.RCFT copy score, 17.RCFT copy time, 18.SVLT recall trial1, 19.SVLT recall trial2, 20.SVLT recall trial3, 21.SVLT total recall, 22.SVLT delayed recall, 23.SVLT recognition discriminability index, 24.RCFT immediate recall, 25.RCFT delayed recall, 26.RCFT recognition discriminability index, 27.Motor impersistence(o), 28.Contrasting program(o), 29.Go No Go(o), 30.Alternating hand movement(o), 31.Alternating square and triangle(o), 32.Luria loop(o), 33.COWAT animal, 34.COWAT supermarket, 35.COWAT phonemic total score, 36.StroopTest Word reading correct, 37.StroopTest Word reading error, 38.StroopTest Color reading correct, 39.StroopTest Color reading error, 40.MMSE orientation to time, 41.MMSE orientation to place, 42.MMSE Registation, 43.MMSE attention and calculation, 44.MMSE recall, 45.MMSE language, 46.MMSE drawing, 47.Outcome

"(o)" was marked on the features of ordinal scale. SNSB, Seoul Neuropsychological Screening Battery; BNT, Boston Naming Test; RCFT, ReyOsterrieth Complex Figure Test; SVLT, Seoul Verbal Learning Test; COWAT, Controlled Oral Word Association Test; MMSE, Mini Mental Status Examination, RFE, Recursive Feature Elimination by ' $n b_{-}$classes $=2$ '. This measure was adopted to ensure consistency when predicting $\mathrm{NC}, \mathrm{MCI}$ and $\mathrm{ADD}$.

This artificial neural network consisted of three layers: an input layer, an output layer, and a hidden layer. To improve the prediction, we performed Xavier method of weight initialization, and the cost was calculated via a cross entropy and minimized by means of the Adam optimizer method (Additional file 1: Table S3). The softmax classifier is used to predict the output labels. The dropout rate was 0.9 , therefore 9 of 10 weights were connected to the next layer to prevent overfittings. Model training was performed with the datasets featuring all 46 variables (Table 1). Ten-fold cross-validation tests of the 2-way classifications using the training dataset was performed with KFold function (Additional file 1: Table S5). After validating the algorithm using 10-fold cross-validation within training datasets, we apply the algorithm 10 times on the test dataset. We thereby obtained the average of prediction accuracy, sensitivity, specificity, positive predictive value and negative predictive value of the algorithm by repeating the process 10 times which obtained from the test data.

This process was performed in both balanced dataset and clinic-based dataset.

Test B. differentiate subjects with Normal cognition and mild cognitive impairment

The accuracy of predicting MCI was evaluated using the balanced dataset and clinic-based dataset. The previous 
algorithm to differentiate $\mathrm{NC}$ and $\mathrm{CI}$ was used $(A)$. Training and ten-fold cross-validation test were performed also with two datasets featuring the 46 variables, and we obtained the 10 times mean prediction accuracy from the test datasets. The sensitivity, specificity, positive predictive value, and negative predictive value of the algorithm were obtained.

Test C. differentiate subjects with normal cognition, $\mathrm{MCl}$, and ADD (Additional file 1: Table S4)

The same datasets used in the Test A, but the outcome included all three outcomes (NC, MCI and ADD). These data were randomly split into training (75\%) and test (25\%) datasets. An artificial neural network also consisted of one input layer, one output layer, and one hidden layers. 'one_hot encoding' was used for differentiating subjects with NC, MCI and ADD by 'nb_classes $=3$ '. The cost was calculated via a cross entropy and minimized by means of the Adam optimizer (Additional file 1: Table S4). The dropout rate was 0.9 . We trained and tested this algorithm to predict either NC, MCI, or ADD 10 times and measured the mean accuracy of each using the test datasets. Ten-fold cross-validation tests of the 3-way classifications using the training dataset was also performed with KFold function (Additional file 1: Table S6).

To determine the extent to which features of the SNSB contribute to acceptable accuracy in predicting target outcome, we listed the 46 variables in order of their contribution using Recursive Feature Elimination (RFE) with a logistic regression algorithm via python 3.6 and its libraries, NumPy, and Pandas (Additional file 1: Table S2 and S3, modified from Feature Selection For Machine Learning in Python, https://machinelearningmastery. com/feature-selection-machine-learning-python/). The algorithms of Test $\mathrm{A}$ and $\mathrm{C}$ were evaluated by adding features one by one, including age and education year, until the accuracy of predicting the target outcome was greater than $90 \%$.

\section{Results}

To predict CI, MCI or ADD, the logistic regression and various layers of the neural network algorithms were compared before applying it on the SNSB features, and a 3-layer neural network with 0.9 drop-out rate was used (Fig. 2 and Additional file 1: Table S1 -S4).

Ten-fold cross-validations were performed using balanced and clinic-based training dataset. The score of cross validation in 2-way (CI vs $\mathrm{NC}$ ) and 3-way (ADD vs MCI vs NC) classification were $96.44 \pm 0.96 \%$ and $95.89 \pm 0.99 \%$ in using balaced dataset; and were $97.51 \pm 0.40 \%$ and $97.01 \pm 0.54 \%$ in clinic based dataset (Table 2).

The first experiment explored whether the algorithm could accurately predict cognitive impairment from a 2way classification dataset (CI and NC, Test A) (Table 3).
The 10 times mean accuracies in identifying $\mathrm{CI}$ in the test datasets from the balanced dataset and the clinicbased dataset achieved by $96.66 \pm 0.52 \%$ and $97.23 \pm$ $0.32 \%$. Their sensitivities were 91.5 and $97.4 \%$; and the specificities were 96.8 and $95.2 \%$. When the accuracies in predicting $\mathrm{MCI}$ from $\mathrm{NC}$ were evaluated, the mean accuracies of the balanced dataset and the clinic-based dataset were $96.60 \pm 0.45$ and $97.05 \pm 0.38 \%$. They showed over $95 \%$ of sensitivity and specificity.

The last experiment (Test $\mathrm{C}$ ) was used to assess the accuracy of the algorithm when predicting one of the three outcomes (NC, MCI or AD); the mean accuracy of the balanced dataset and the clinic-based dataset were $95.49 \pm 0.53$ and $96.34 \pm 1.03 \%$ (Table 3).

In 2-way ( $\mathrm{NC}$ or $\mathrm{CI}$ ) and 3-way (NC, MCI and ADD) classification, the order of 46 variables in their contribution were evaluated using the Recursive Feature Elimination (RFE). The following 2 extracted variables contributed the most to predicting the target outcome in order of ranking: 'MMSE_orientation_to_time' and 'MMSE_recall', which are memory related features. The next features contributing the outcome of the predictions were shown in Fig. 3. When 'MMSE_orientation to_time', the most contributor, was added, with age and education years, the accuracy to predict cognitive impairment in the balanced dataset was $73.7 \%$, and $\mathrm{MCI}$ or ADD was $61.1 \%$. Then, an MMSE_recall was added, and the accuracy increased to 77.7 and $64.5 \%$. When using the clinic-based dataset, the first feature was added, and the prediction of cognitive impairment was $78.3 \%$ and MCI or ADD was $60.1 \%$. The second feature was added to increase it to 80.2 and $63.5 \%$. However, when the number of features was 6 , the accuracy was more than $80 \%$ in prediction of the cognitive impairment. As the number of features increased to 10 and 12, respectively, 2-way and 3-way classification showed more than 90\% accuracy respectively (Fig. 3 ).

\section{Discussion}

As an exploratory study, we first examined the logistic regression and various layers of neural network algorithms. Neural network algorithm was better than logistic regression. Among them, the 3-layer neural network algorithm was the best (Fig. 2). The accuracy of 2-way classification ( $\mathrm{NC}$ vs $\mathrm{CI}$ ) in the balanced dataset using the logistic regression that is commonly used for classification was $85.9 \%$, but 3 -way classification ( $\mathrm{NC}$ vs $\mathrm{MCI}$ vs ADD) was only $79.0 \%$. Compared with the logistic regression, the neural network was superior to predict the target outcome. By empirically changing the parameters one by one, we selected the parameters that showed the best performance. Particularly, when comparing 3, 4, 5, and 6-layer of the neural network, the best prediction was made in the 3-layer neural network. The dropout 


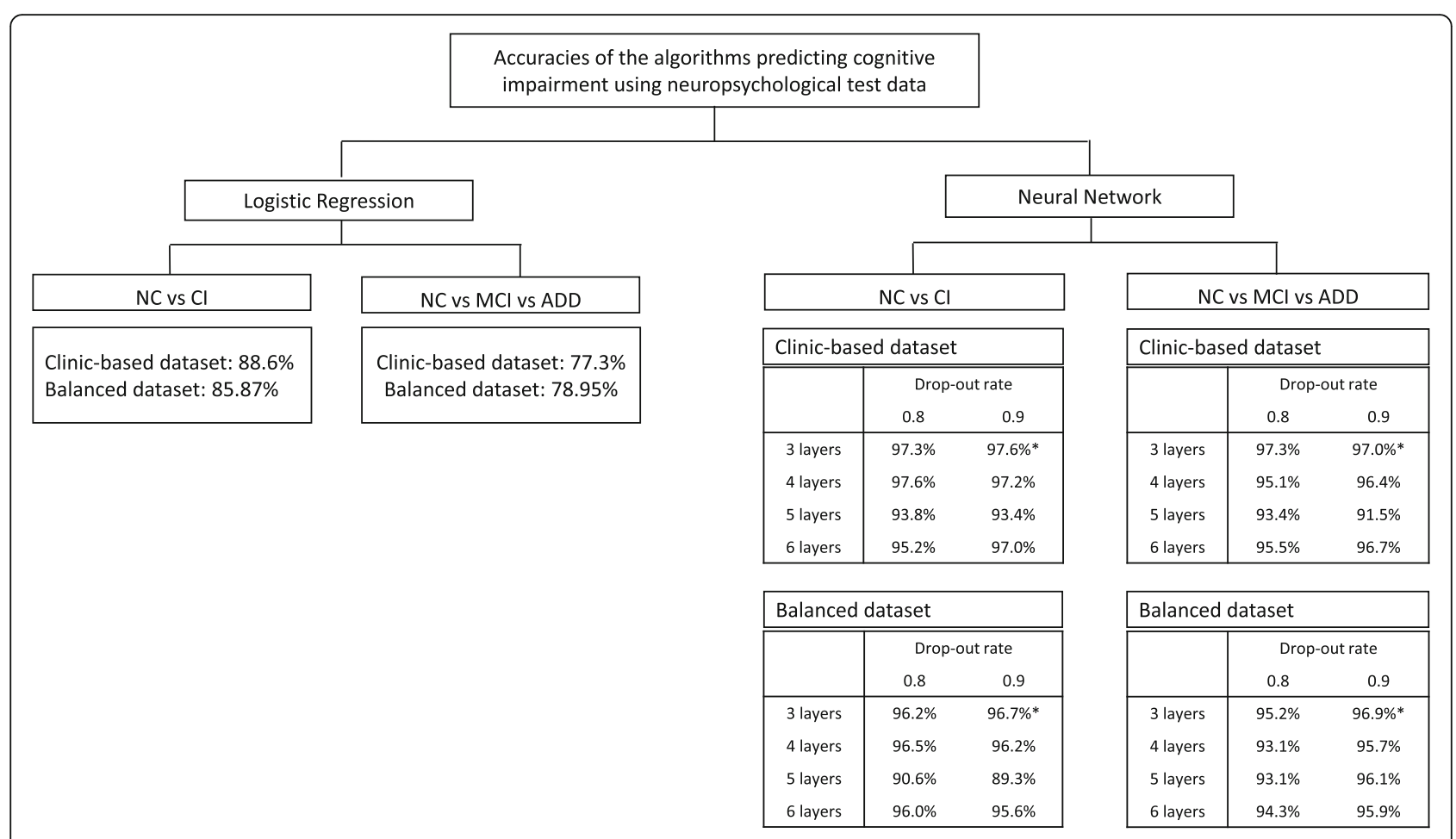

Fig. 2 Comparison of accuracies in Logistic Regression and various layers of Neural-Network algorithm

probability 0.8 and 0.9 were acceptable, 0.9 of which was chosen (Fig. 2), and the learning rate was 0.01 . Therefore, we did supervised-learning with the 3-layer neural network in this study (Additional file 1: Table S3, S4), and found over $95 \%$ accuracy of 2-way classification and of 3-way classification (Table 3). The sensitivity and specificity of the 3-layer of neural network for the detection of CI in the balanced dataset were 96.0 and $96.8 \%$, and MCI were 96.0 and $97.4 \%$. The 2-way classification algorithms showed high enough sensitivity and specificity more than $85 \%$, which is generally acceptable new biomarkers for a neurodegenerative disorder such as $\mathrm{AD}$ or Parkinson's disease $[26,27]$, which are usuable as a reference tool [28].

There would be a concern that it may fall into a circularity problem in predicting cognitive impairment. There are two points to keep in mind when applying artificial intelligence algorithms. The first is to allow the algorithm to take over the troublesome task for human, and the second is to do better than we can do what we can't do. The purpose of building algorithm in this study was to aid clinicians to sorting out patients with cognitive impairment from large number of cases thereby expert judges can focus on cases which require medical attention. The authors would like to have algorithms make judgments similar to those of humans when using neuropsychological tests. The algorithms only need to mimic what neuropsychologist do. However, if the aim was to make accurate diagnoses beyond human capabilities, like predicting AD by only looking at brain MRI, then the study should consider circularity issues. For more accurate diagnosis by the AI algorithm, the MRI features should not contaminate the outcome of clinical diagnosis. Since the neuropsychological tests inform the presence of objective cognitive impairment, they can necessarily influence clinical diagnosis and cannot escape circularity problem. The disease state,

Table 2 Ten-fold cross-validation test results using balanced and clinic-based dataset

\begin{tabular}{lllll}
\hline & & Minimum(\%) & Maximum(\%) & Mean \pm SD(\%) \\
\hline Balanced dataset & Cl vs NC & 95.03 & 97.93 & $96.44 \pm 0.96$ \\
& MCI vs NC & 94.82 & 97.31 & $96.11 \pm 0.69$ \\
Clinic-based dataset & ADD vs MCl vs NC & 93.66 & 96.82 & $95.89 \pm 0.99$ \\
& Cl vs NC & 96.96 & 98.21 & $97.51 \pm 0.40$ \\
& MCI vs NC & 96.53 & 98.84 & $97.27 \pm 0.67$ \\
& ADD vs MCl vs NC & 96.34 & 97.86 & $97.01 \pm 0.54$ \\
\hline
\end{tabular}


Table 3 Prediction accuracy of the neural network algorithm using the neuropsychological screening test dataset

\begin{tabular}{|c|c|c|c|c|c|c|c|c|}
\hline & Prediction & Number of subjects & $\begin{array}{l}\text { Accuracy of } 10 \text { trials } \\
\text { (mean } \pm \text { SD\%) }\end{array}$ & SE(\%) & $\mathrm{SP}(\%)$ & PPV(\%) & NPV(\%) & $A \cup C$ \\
\hline \multirow[t]{3}{*}{ Balanced dataset } & $\mathrm{Cl}$ vs NC & $3231: 3217$ & $96.66 \pm 0.52$ & 96.0 & 96.8 & 97.0 & 95.8 & 0.964 \\
\hline & $\mathrm{MCl}$ vs NC & $3217: 3217$ & $96.60 \pm 0.45$ & 96.0 & 97.4 & 97.6 & 95.6 & 0.967 \\
\hline & ADD vs $\mathrm{MCl}$ vs $\mathrm{NC}$ & 3235: 3217: 3217 & $95.49 \pm 0.53$ & & & & & \\
\hline \multirow[t]{3}{*}{ Clinic-based dataset } & $\mathrm{Cl}$ vs NC & 11,709: 3217 & $97.23 \pm 0.32$ & 97.4 & 95.2 & 98.6 & 91.3 & 0.963 \\
\hline & $\mathrm{MCl}$ vs NC & 6002: 3217 & $97.05 \pm 0.38$ & 97.5 & 96.4 & 98.1 & 94.8 & 0.968 \\
\hline & ADD vs $\mathrm{MCl}$ vs NC & 5707: 6002: 3217 & $96.34 \pm 1.03$ & & & & & \\
\hline
\end{tabular}

SD Standard deviation, SE Sensitivity, SP Specificity, PPV Positive predictive value, NPV Negative predictive value, AUC Area under the curve, CI Cognitive impairment, $\mathrm{NC}$ Normal cognition, $\mathrm{MCl}$ Mild cognitive impairment

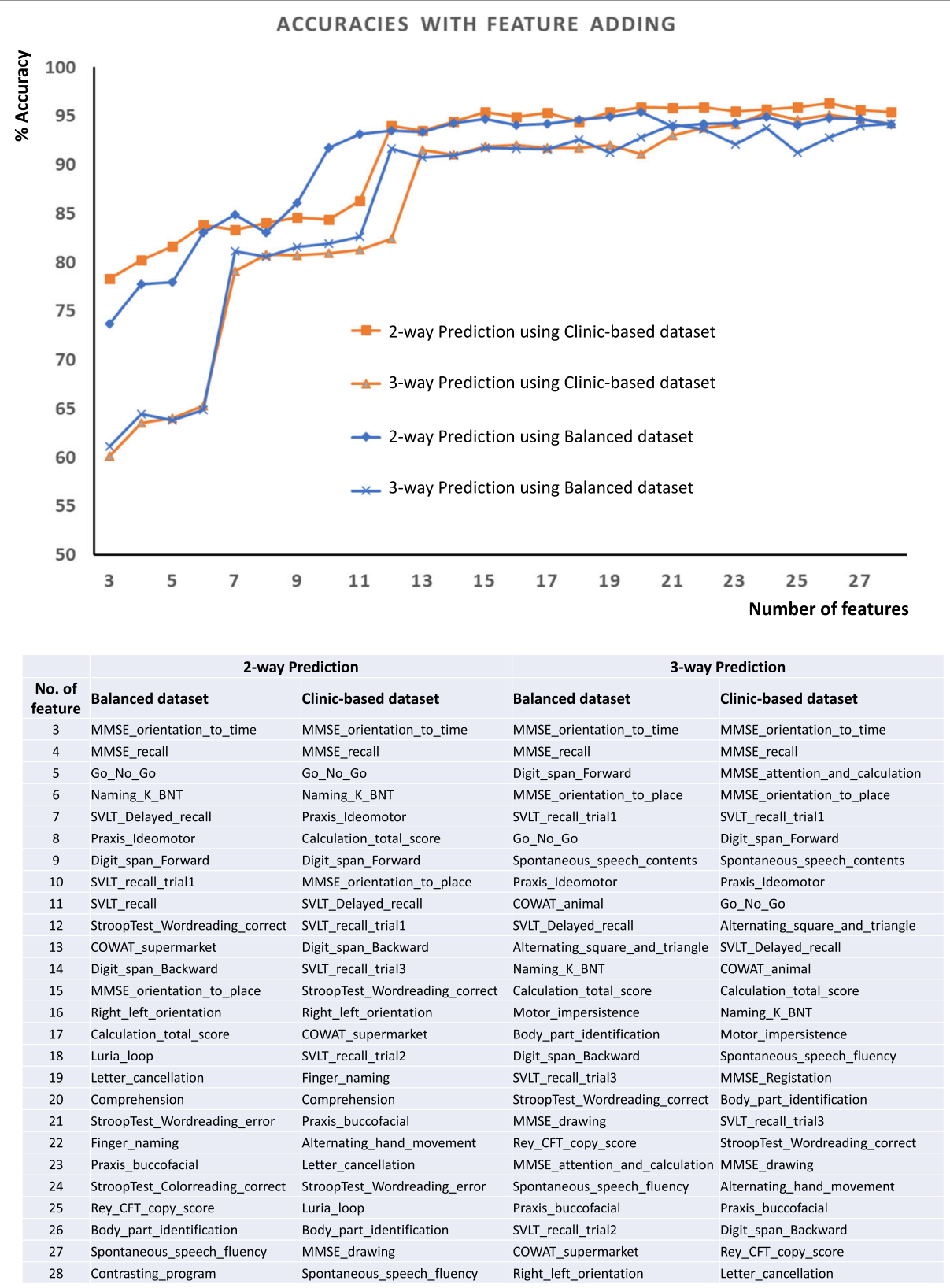

Fig. 3 Accuracy increment with adding feature one by one 
outcome feature of the dataset, was diagnosed finally depend on clinical decisions with considering cognitive function. While $\mathrm{NC}$ and $\mathrm{CI}$ can be classified by feature of neuropsychological test, $\mathrm{MCI}$ and $\mathrm{AD}$ dementia among patients with cognitive impairment are determined by presence of disability in daily life, which is not included as predictor in the algorithm of this study $[4,5,28]$.

There are some studies having similarities in classifying patients with $\mathrm{AD}$ and optimizing features of neuropsychological test data to reduce the required features to predict target outcomes $[9,29]$. They used the CDR score, severity of cognitive impairment, as criteria of categorization and used stratified randomization of subjects into three categories of CDR 0, 0.5 and 1 . However, we classified subjects into $\mathrm{NC}, \mathrm{MCI}$ and ADD by clinical decision rather than CDR, which was an different approach. Patient with CDR 0.5 could be an early stage $\mathrm{AD}$ or MCI, but not exclude other cause of dementia. More precisely, NC in this study was 'subjective cognitive declines' who visited the hospital with complaints about cognitive dysfunction and were judged normal in neuropsychological tests [30]. MCI is a condition that lies on a continuum between healthy aging and dementia [31]. Neuropsychological test, conducted by trained psychologists, is one of the information to be considered for the final diagnosis by clinicians taking into account not only neuropsychological data but also several laboratory tests and medical history obtained from the patients and their caregivers. As the algorithm lacked input from clinicians and only employed neuropsychological test data, the accuracy of predicting one out of three conditions was expected to be inevitably lower. The relatively superior accuracy of 2-way classification in small samples has also been demonstrated by prior machine-learning research [12]. It is interesting to note that using machine learning with neuropsychological data alone could distinguish accurately between MCI and ADD which requires a clinical decision. Future research can confirm the finding.

In clinic-based dataset, there were imbalances of subjects in both classifications; 2-way classification was $3217 \mathrm{NC}$ vs $11,709 \mathrm{CI}$, and 3-way classification was 3217 $\mathrm{NC}$ vs $6002 \mathrm{MCI}$ vs 5707 ADD. Although we did not perform stratification randomization, we think that it showed relatively high prediction accuracy and low variability for each trial because there was a larger dataset (Table 3). In a study with a relatively small number of subjects, stratified randomization can exclude differences by chance and can increase the reliability of the results [32]. However, we did not stratified randomization to use all possible neuropsychological data, which would be an almost real prevalence of patients visiting the hospital who want to be assessed for cognitive impairment. This study was not intended to assess neuropsychological characteristics of cognitive function nor to apply the algorithm to screening tools for community-based populations. We suggest it can be possible used as a reference when clinicians read neuropsychological tests got from hospital-based patients.

The algorithm of CI vs NC and $\mathrm{MCI}$ vs NC using 1012 variables exhibited higher accuracy of prediction; there are possible implications from a dementia screening perspective. The features of the neuropsychological tests were listed in order of their contribution to the outcome using RFE (Fig. 3). Six figures with age and educational duration predicted outcomes more than $80 \%$ of the accuracy, and 12 features increased to more than 90\% of the accuracy: an adequate level for machinelearning feasibility. Variable selection in machine learning is widely used to avoid data over-fit, provide faster and more effective models, and improve the accuracy of classification. Variable reduction using statistic algorithms provides the minimum subset of variables necessary for the classification model and saves time and cost for evaluation [33]. Weakley et al. conducted a study to determine the fewest number of clinical measures required for differentiating older patients with dementia from their healthy counterparts. Their results showed that as few as two to nine variables may be sufficient to obtain a clinically useful classification model [12]. It is also necessary to evaluate the value of the cognitive impairment screening test algorithm using reduced variables of the neuropsychological test.

Kang et al. compared the neuropsychological profiles between $\mathrm{AD}$ and mixed dementia using CREDOS dataset which target population partly overlaps with ours [34]. The current study used larger dataset and targeted to distinguish $\mathrm{MCI}$ and dementia in the spectrum of $\mathrm{AD}$ using machine learning algorithms. We tested the algorithms in the two dataset, clinic-based and balanced datasets. Although the 2-way classification (NC and $\mathrm{MCI}+\mathrm{ADD}$ ) was imbalanced in clinic-based dataset, the repeated trials showed low variability of accuracy and high specificity, and similar accuracies to the balanced dataset.

The present study is subject to several limitations. First, the model is only applicable to differentiate cognitive states and cannot predict the temporal stage or prognosis. Second, the dementia group only includes ADD; therefore, the model does not apply to other subtypes of dementia. Therefore more research is needed on these two respects.

The purpose of this study was to evaluate a neural network algorithm that could predict $\mathrm{NC}, \mathrm{MCI}$, and $\mathrm{ADD}$ from 46-features of formal neuropsychological data obtained from the hospitals. Our results indicated that 46variable algorithm achieved acceptable accuracy, sensitivity and specificity. We also identified the order of 
contributions of the features that predict cognitive impairment, and approximately 12-13 from 46 features played an important role in acceptable accurate prediction.

\section{Conclusions}

We trained and tested a machine-learning algorithm model using a large set of neuropsychological test data to distinguish between normal and cognitively impaired patients and suggest its potential use as a reference when clinicians see the neuropsychological test. Future studies are required, however, to yield an algorithm that can predict the progressor with a higher level of classification-efficiency that is capable of use in clinical settings, and can predict other causes of cognitive impairment.

\section{Supplementary information}

Supplementary information accompanies this paper at https://doi.org/10 1186/s12911-019-0974-x.

Additional file 1 Table S1. Logistic Regression algorithm to differentiate cognitive impairment from normal cognition using the neuropsychological test dataset. Table S2. Logistic Regression algorithm to differentiate mild cognitive impairment and Alzheimer's disease dementia from normal cognition using the neuropsychological test dataset. Table S3. Neural network algorithm to differentiate cognitive impairment from normal cognition using using the neuropsychological test dataset. Table S4. Neural network algorithm to differentiate mild cognitive impairment and Alzheimer's disease dementia from normal cognition using the neuropsychological test dataset. Table S5. Algorithm for 10-fold cross validation of 2-way classification (cognitive impairment from normal cognition) using the neuropsychological test dataset. Table S6. Algorithm for 10-fold cross validation of 3-way classification (mild cognitive impairment or Alzheimer's disease dementia from normal cognition). Table S7. Feature extraction with Recursive Feature Elimination from variables of the neuropsychological test (modified from Feature Selection for Machine Learning in Python, https://machinelearningmastery.com/featureselection-machine-learning-python/).

\section{Abbreviations}

AD: Alzheimer's disease; ADD: Alzheimer's Disease Dementia; ADL: Activity of daily living; BDSNUH: Bungdang Seoul National University Hospital; CAUH: Chung-Ang University Hospital; CERAD: Consortium to Establish a Registry for Alzheimer's disease; Cl: Cognitive impairment;

COWAT: Controlled oral word association test; CREDOS: The Clinical Research Center for Dementia of South Korea; K_MMSE: Korean version of the MiniMental Status Examination; MCl: Mild cognitive impairment; NC: Normal Cognition; NPTs: Neuropsychological tests; RCFT: Rey-Complex figure test; RFE: Recursive Feature Elimination; ROC: Receiver operator characteristic; SNSB: Seoul Neuropsychological Screening Battery; SVLT: Seoul verbal learning test

\section{Acknowledgements}

Not Applicable.

\section{Authors' contributions}

MJK and YCY contributed toward data analysis and drafting. MJK, SYK', DLN, BCK, DWY, EK, HRN, HJH, JL, JHK, KHP, KWP, SH, SYK², SJY, BY, SWS, SYM, YSY, YSS, MJB, JHJ, SHC and YCY contributed toward collecting data, revising the paper and agree to be accountable for all aspects of the work. All authors read and approved the final version of the manuscript. 'SangYun Kim, ${ }^{2}$ Seong Yoon Kim.

\section{Funding}

The publication costs, design of the study, data management and writing the manuscript for this article were supported by the Ministry of Education of the Republic of Korea and the National Research Foundation of Korea (NRF-2017S1A6A3A01078538), Korea Ministry of Health \& Welfare, and from the Original Technology Research Program for Brain Science through the National Research Foundation of Korea funded by the Korean Government (MSIP; No. 2014M3C7A1064752).

\section{Availability of data and materials}

The datasets used and/or analyzed in this study are available from the CREDOS (request the data through http://public.crcd.or.kr/) and are available from the corresponding author or Seong Hye Choi, PI of CREDOS (seonghye@inha.ac.kr).

Ethics approval and consent to participate

This study was approved by the institutional review boards of Chung-Ang University Hospital (IRB number C2012049(744)).

\section{Consent for publication}

Not Applicable.

\section{Competing interests}

The authors declare that they have no competing interests.

\section{Author details}

'Department of Neurology, Seoul National University College of Medicine \& Seoul National University Bundang Hospital, Seoul, South Korea. ${ }^{2}$ Department of Neurology, Veterans Health Service Medical Center, Seoul, South Korea. ${ }^{3}$ Department of Neurology, Samsung Medical Center, Sungkyunkwan University School of Medicine, Seoul, South Korea. ${ }^{4}$ Department of Neurology, Chonnam National University Medical School, Gwangju, South Korea. ${ }^{5}$ Department of Neurology, College of Medicine, The Catholic University of Korea, Seoul, South Korea. ${ }^{6}$ Department of Neurology, Pusan National University Hospital, Pusan National University School of Medicine and Medical Research Institute, Busan, South Korea. ${ }^{7}$ The Brain Fitness Center, Bobath Memorial Hospital, Seongnam, South Korea. ${ }^{8}$ Department of Neurology, Myongji Hospital, Hanyang University College of Medicine, Goyang, South Korea. ${ }^{9}$ Department of Neurology, University of Ulsan College of Medicine, Asan Medical Center, Seoul, South Korea. ${ }^{10}$ Department of Neurology, Dementia Center, Ilsan Hospital, National Health Insurance Service, Goyang, South Korea. ${ }^{11}$ Department of Neurology, College of Medicine, Gachon University Gil Hospital, Incheon, South Korea.

${ }^{12}$ Department of Neurology, Dong-A University College of Medicine and Institute of Convergence Bio-Health, Busan, South Korea. ${ }^{13}$ Department of Neurology, Konkuk University Medical Center, Seoul, South Korea. ${ }^{14}$ Department of Psychiatry, University of Ulsan College of Medicine, Asan Medical Center, Seoul, South Korea. ${ }^{15}$ Department of Neurology, Eulji University College of Medicine, Daejeon, South Korea. ${ }^{16}$ Department of Neurology, Konyang University Hospital, College of Medicine, Konyang University, Daejeon, South Korea. ${ }^{17}$ Department of Neurology, Ajou University School of Medicine, Suwon, South Korea. ${ }^{18}$ Department of Neurology, Eunpyeong St. Mary's Hospital, College of Medicine, The Catholic University of Korea, Seoul, South Korea. ${ }^{19}$ Department of Neurology, Ewha Womans University School of Medicine, Seoul, South Korea. ${ }^{20}$ Department of Neurology, Inha University School of Medicine, Incheon, South Korea. ${ }^{21}$ Department of Neurology, College of Medicine, Chung-Ang University, Seoul, South Korea.

Received: 18 April 2019 Accepted: 8 November 2019

Published online: 21 November 2019

References

1. Cheng YW, Chen TF, Chiu MJ. From mild cognitive impairment to subjective cognitive decline: conceptual and methodological evolution. Neuropsychiatr Dis Treat. 2017;13:491-8.

2. Peters F, Villeneuve S, Belleville S. Predicting progression to dementia in elderly subjects with mild cognitive impairment using both cognitive and neuroimaging predictors. J Alzheimers Dis. 2014;38(2):307-18.

3. Baerresen KM, Miller KJ, Hanson ER, Miller JS, Dye RV, Hartman RE, Vermeersch D, Small GW. Neuropsychological tests for predicting cognitive decline in older adults. Neurodegener Dis Manag. 2015;5(3):191-201.

4. McKhann GM, Knopman DS, Chertkow H, Hyman BT, Jack CR Jr, Kawas CH, Klunk WE, Koroshetz WJ, Manly JJ, Mayeux R, et al. The diagnosis of 
dementia due to Alzheimer's disease: recommendations from the Nationa Institute on Aging-Alzheimer's Association workgroups on diagnostic guidelines for Alzheimer's disease. Alzheimers Dement. 2011;7(3):263-9.

5. McKhann G, Drachman D, Folstein M, Katzman R, Price D, Stadlan EM. Clinical diagnosis of Alzheimer's disease: report of the NINCDS-ADRDA work group under the auspices of Department of Health and Human Services Task Force on Alzheimer's disease. Neurology. 1984;34(7):939-44.

6. Beier M, Hughes AJ, Williams MW, Gromisch ES. Brief and cost-effective tool for assessing verbal learning in multiple sclerosis: comparison of the Rey auditory verbal learning test (RAVLT) to the California verbal learning test - ॥ (CVLT-II). J Neurol Sci. 2019;400:104-9.

7. Muller S, Herde L, Preische O, Zeller A, Heymann P, Robens S, Elbing U, Laske C. Diagnostic value of digital clock drawing test in comparison with CERAD neuropsychological battery total score for discrimination of patients in the early course of Alzheimer's disease from healthy individuals. Sci Rep. 2019;9(1):3543

8. Deo RC. Machine learning in medicine. Circulation. 2015;132(20):1920-30.

9. Battista P, Salvatore C, Castiglioni I. Optimizing neuropsychological assessments for cognitive, behavioral, and functional impairment classification: a machine learning study. Behav Neurol. 2017;2017:1850909.

10. Han SH, Kim KW, Kim S, Youn YC. Artificial neural network: understanding the basic concepts without mathematics. Dement Neurocogn Disord. 2018; 17(3):83-9.

11. Gurevich P, Stuke H, Kastrup A, Stuke H, Hildebrandt H. Neuropsychological testing and machine learning distinguish Alzheimer's disease from other causes for cognitive impairment. Front Aging Neurosci. 2017;9:114.

12. Weakley A, Williams JA, Schmitter-Edgecombe M, Cook DJ. Neuropsychological test selection for cognitive impairment classification: a machine learning approach. J Clin Exp Neuropsychol. 2015;37(9):899-916.

13. Moradi E, Pepe A, Gaser C, Huttunen H, Tohka J. Alzheimer's disease neuroimaging I: machine learning framework for early MRI-based Alzheimer's conversion prediction in MCl subjects. Neuroimage. 2015;104: 398-412.

14. Youn YC, Choi SH, Shin HW, Kim KW, Jang JW, Jung JJ, Hsiung GR, Kim S. Detection of cognitive impairment using a machine-learning algorithm. Neuropsychiatr Dis Treat. 2018;14:2939-45.

15. Choi HJ, Lee DY, Seo EH, Jo MK, Sohn BK, Choe YM, Byun MS, Kim JW, Kim SG, Yoon JC, et al. A normative study of the digit span in an educationally diverse elderly population. Psychiatry Investig. 2014;11(1):39-43.

16. Kim H, Na DL. Normative data on the Korean version of the Boston naming test. J Clin Exp Neuropsychol. 1999;21(1):127-33.

17. Shin MS, Park SY, Park SR, Seol SH, Kwon JS. Clinical and empirical applications of the Rey-Osterrieth complex figure test. Nat Protoc. 2006;1(2):892-9.

18. Baek MJ, Kim HJ, Kim S. Comparison between the story recall test and the word-list learning test in Korean patients with mild cognitive impairment and early stage of Alzheimer's disease. J Clin Exp Neuropsychol. 2012;34(4): 396-404.

19. Park HK, Na DL, Han SH, Kim JY, Cheong HK, Kim SY, Kim SY, Hong CH, Kim DK, Ku BD, et al. Clinical characteristics of a nationwide hospital-based registry of mild-to-moderate Alzheimer's disease patients in Korea: a CREDOS (clinical research Center for Dementia of South Korea) study. J Korean Med Sci. 2011;26(9):1219-26.

20. Ye BS, Seo SW, Lee Y, Kim SY, Choi SH, Lee YM, Kim DH, Han HJ, Na DL, Kim EJ. Neuropsychological performance and conversion to Alzheimer's disease in early- compared to late-onset amnestic mild cognitive impairment: CREDOS study. Dement Geriatr Cogn Disord. 2012;34(3-4):156-66.

21. Chang KJ, Lee S, Lee Y, Lee KS, Back JH, Jung YK, Lim KY, Noh JS, Kim HC, Roh HW, et al. Severity of white matter Hyperintensities and length of hospital stay in patients with cognitive impairment: a CREDOS (clinical research Center for Dementia of South Korea) study. J Alzheimers Dis. 2015; 46(3):719-26.

22. Park HK, Choi SH, Park SA, Kim HJ, Lee Y, Han SH, Kim EJ, Kim BC, Han HJ, Moon SY, et al. Cognitive profiles and neuropsychiatric symptoms in Korean early-onset Alzheimer's disease patients: a CREDOS study. J Alzheimers Dis. 2015;44(2):661-73.

23. Roh HW, Hong CH, Lee Y, Lee KS, Chang KJ, Kang DR, Lee JD, Choi SH, Kim SY, Na DL, et al. Clinical conversion or reversion of mild cognitive impairment in community versus hospital based studies: GDEMCIS (Gwangju dementia and mild cognitive impairment study) and CREDOS (clinical research Center for Dementia of South Korea). J Alzheimers Dis. 2016;53(2):463-73.
24. Choi SH, Kim S, Han SH, Na DL, Kim DK, Cheong HK, Lee JH, Kim SY, Hong $\mathrm{CH}$, Moon SY, et al. Neurologic signs in relation to cognitive function in subcortical ischemic vascular dementia: a CREDOS (clinical research Center for Dementia of South Korea) study. Neurol Sci. 2012;33(4):839-46.

25. Rampasek L, Goldenberg A. TensorFlow: Biology's gateway to deep learning? Cell Syst. 2016;2(1):12-4.

26. Prashanth R, Dutta Roy S, Mandal PK, Ghosh S. High-accuracy detection of early Parkinson's disease through multimodal features and machine learning. Int J Med Inform. 2016;90:13-21.

27. Consensus report of the Working Group on. "Molecular and Biochemical Markers of Alzheimer's Disease". The Ronald and Nancy Reagan Research Institute of the Alzheimer's Association and the National Institute on Aging Working Group. Neurobiol Aging. 1998;19(2):109-16.

28. Ballard C, Gauthier S, Corbett A, Brayne C, Aarsland D, Jones E. Alzheimer's disease. Lancet. 2011;377(9770):1019-31.

29. Salvatore C, Cerasa A, Castiglioni I. MRI characterizes the progressive course of $\mathrm{AD}$ and predicts conversion to Alzheimer's dementia 24 months before probable diagnosis. Front Aging Neurosci. 2018;10:135.

30. Kuhn E, Moulinet I, Perrotin A, La Joie R, Landeau B, Tomadesso C, Bejanin A, Sherif S, De La Sayette V, Desgranges B, et al. Cross-sectional and longitudinal characterization of SCD patients recruited from the community versus from a memory clinic: subjective cognitive decline, psychoaffective factors, cognitive performances, and atrophy progression over time. Alzheimers Res Ther. 2019;11(1):61.

31. Feldman HH, Jacova C. Mild cognitive impairment. Am J Geriatr Psychiatry. 2005:13(8):645-55

32. Saghaei M. An overview of randomization and minimization programs for randomized clinical trials. J Med Signals Sens. 2011;1(1):55-61.

33. Saeys $Y$, Inza I, Larranaga P. A review of feature selection techniques in bioinformatics. Bioinformatics. 2007;23(19):2507-17.

34. Kang HS, Kwon JH, Kim S, Na DL, Kim SY, Lee JH, Han SH, Choi SH, Kim S, Carroll BJ, et al. Comparison of neuropsychological profiles in patients with Alzheimer's disease and mixed dementia. J Neurol Sci. 2016:369:134-8.

\section{Publisher's Note}

Springer Nature remains neutral with regard to jurisdictional claims in published maps and institutional affiliations.
Ready to submit your research? Choose BMC and benefit from:

- fast, convenient online submission

- thorough peer review by experienced researchers in your field

- rapid publication on acceptance

- support for research data, including large and complex data types

- gold Open Access which fosters wider collaboration and increased citations

- maximum visibility for your research: over $100 \mathrm{M}$ website views per year

At $\mathrm{BMC}$, research is always in progress.

Learn more biomedcentral.com/submissions 